Check for updates

Cite this: Soft Matter, 2018, 14,8182

Received 25th May 2018 , Accepted 19th August 2018

DOI: $10.1039 / c 8 s m 01082 j$

rsc.li/soft-matter-journal

\section{Droplet impact dynamics on textiles}

\author{
Gannian Zhang, (D) a Miguel A. Quetzeri-Santiago, ${ }^{a}$ Corinne A. Stone, ${ }^{b}$ \\ Lorenzo Botto (D) and J. Rafael Castrejón-Pita (D)*a
}

\section{Introduction}

The impact of liquid droplets on solid surfaces is ubiquitous in nature, such as raindrops striking the surfaces of soil ${ }^{1}$ and plants. $^{2}$ In industrial environments, these impact dynamics are important in a wide range of applications, such as the design of functional surfaces for self-cleaning, ${ }^{3,4}$ ice repulsion, ${ }^{5,6}$ and the manufacturing of smart ${ }^{7}$ and protective ${ }^{8}$ clothing. Historically, researchers have extensively studied droplet spreading and receding, ${ }^{9}$ bouncing and splashing ${ }^{10-12}$ on smooth and rough solid surfaces, and on complex and smart substrates. ${ }^{13}$ Symmetric droplet splitting ${ }^{2,14}$ and pancake bouncing ${ }^{13}$ have been observed on solid surfaces with superhydrophobic ridges and micro-posts. More recently some authors have studied the impact of droplets on a sieve ${ }^{15}$ and on rigid metallic meshes ${ }^{16,38}$ concluding that the impact dynamics on these substrates depends on the impact speed and the substrate characteristics. However, the impact of droplets on textiles has received little attention. Previous studies on textiles have been confined to the qualitative comparison of bloodstain patterns following blood droplet impact on different textiles ${ }^{17}$ and the estimation of the textiles' impact stresses. ${ }^{18}$

\footnotetext{
${ }^{a}$ School of Engineering and Materials Science, Queen Mary, University of London, London E1 4NS, UK. E-mail: r.castrejonpita@qmul.ac.uk; Tel: $+44(0) 2078827620$

${ }^{b}$ Defence Science and Technology Laboratory, Porton Down, Salisbury SP4 OJQ, UK
}

Here we study the impact dynamics of liquid droplets on hydrophobic and hydrophilic nylon textiles. We first focus on the droplet spreading behaviour and next we determine the liquid penetration criterion in terms of the textile characteristics.

The impact dynamics of droplets on solid substrates has received much attention due to its relevance in inkjet printing, pesticide and paint spraying, and other aerosol coatings. The fluid mechanical analyses have typically focused on the maximum spreading diameter, and on the splashing behaviour, as these aspects determine the quality of printing in inkjet and the coverage efficiency of sprays. Importantly, it has been found that the maximum spreading ratio $\beta_{\max }=\frac{d_{\max }}{D}$ follows a power law of the form $\beta_{\max } \propto \mathrm{We}^{\alpha}$, where $\alpha$ ranges from 0.2 to $0.5 ; d_{\max }$ and $D$ are the maximum spreading and the initial droplet diameters, respectively, and We is the Weber number (defined as $\mathrm{We}=\frac{\rho v^{2} D}{2 \sigma}, \rho$ is the liquid density, $v$ is the speed of impact, and $\sigma$ is the surface tension). Recent studies ${ }^{19}$ have shown that, in the context of porous media, $\alpha$ is also affected by moisture. Using scaling arguments, Clanet et al. ${ }^{20}$ found that $\beta_{\max }$ is a function of $\mathrm{We}^{1 / 4}$ for both hydrophobic and hydrophilic surfaces for droplet impact in the range $2 \leq \mathrm{We} \leq 900$. In a different work, Lee et $a l^{21}$ found that viscosity also affects $\beta_{\max }$ and proposed a relationship of the form $\left(\beta_{\max }{ }^{2}-\beta_{\text {sessile }}\right)^{1 / 2} \propto \operatorname{Re}^{\frac{1}{5}} f(\mathrm{We})$ based on a first-order Padé approximation, ${ }^{22}$ where $\beta_{\text {sessile }}$ is the spreading ratio at 
rest, $\operatorname{Re}$ is the Reynolds number $\operatorname{Re}=\frac{\rho D v}{2 \mu}, 23$ and $\mu$ is the liquid dynamic viscosity. However, a consensus on whether the surface wettability affects $\beta_{\max }$ has yet to be reached. It has been argued that spreading ratio on surfaces such as stainless steel, glass and paraffin depends on the contact angle $\theta$ and is ruled by a critical Weber (We) number. ${ }^{24,25}$ Experimental data ${ }^{26}$ have shown that for We $<200, \beta_{\max }$ decreases monotonically as a function of $\theta$, while for We $>200, \beta_{\max }$ varies with $\mathrm{We}^{1 / 5}$. In contrast, other evidence from droplet impact on plastic and glass substrates ${ }^{27}$ at We $>200$ has proven that $\theta$ plays no role in $\beta_{\max }$. In the context of solid meshes, ${ }^{20}$ a scaling $d_{\max } \propto$ $D \cdot \mathrm{We}^{1 / 4}$ has recently been found to describe the impact dynamics on non-wettable meshes with a solid fraction $\phi$ no smaller than $70 \%{ }^{16}$

Droplet impact on hydrophobic substrates have also been the focus of many recent studies. The reduction of the contact time between a droplet and a solid substrate during the impact is crucial to the design of self-cleaning and liquid repellent surfaces. Liquid repellency has been achieved by incorporating superhydrophobic micro-ridges ${ }^{14}$ and micro-wires ${ }^{28}$ on smooth surfaces to cause axi-symmetric droplet splitting upon impact. Experiments ${ }^{13}$ have shown that the droplet impact on microarchitectured tapered micro-posts results in either a pancake bouncing or a recoiling-bouncing regime. For straight posts, the liquid menisci are subjected to constant deceleration, resulting in droplet recoiling before bouncing. Other experiments have shown that irregular surface roughness results in diverse bouncing regimes, ranging from conventional spreading to partial bouncing due to droplet pinning on the roughness elements, or to the more rarely observed inverse jetting and bouncing with entrapped air bubbles. ${ }^{29}$ Superhydrophobic micro-patterned substrates can be invaded or flooded by liquid due to impact, evaporation or condensation, ${ }^{30}$ and such "invasion" can be promoted by local extreme roughness protrusions. ${ }^{31}$ Surface patterning leads to partial wetting and pinning of the liquid at the substrate surface. In this transition, a droplet goes from "floating" above the substrate to being impaled at the microposts. ${ }^{32}$ Whether a sessile droplet impales or floats depends on the architecture of the substrate, the cavity size, $\theta$, and the roughness factor. Experimental evidence ${ }^{33}$ has shown that evaporation-based flooding is regulated by the droplet size, the interstices pitch, and the height of the micro-texture, favoring the impalement of small droplets into the micro-texture.

Droplet impact on porous media has received little attention, compared with the abundant research on impermeable solids. Existing papers ${ }^{15,34}$ on permeable solids have focused on droplet impingement normal to the substrates while assuming that the azimuthal (or lateral) spreading encounters no other pore on the solid. Lorenceau and Quéré ${ }^{15}$ studied the impact of millimetresized silicone oil droplets on a wettable solid surface presenting a hole ranging from 200 to $600 \mu \mathrm{m}$ in diameter. A critical velocity above which a droplet penetrates the hole and forms a filament extending beyond the back surface was identified. Under some conditions, this filament elongates and ruptures, generating secondary (or satellite) droplets. A force balance between inertial, capillary and viscous effects produced a scaling relationship connecting the speed of impact, the liquid properties and the pore size. Importantly, experimental data found that wettability of the solid was irrelevant in this case. Delbos $e t a l .^{34}$ studied the impact of millimetre-sized droplets on superhydrophobic submillimetre-sized capillary tubes and found three penetration regimes: no penetration, limited penetration and "slug formation". For an impact at a high speed, most of the droplet volume would penetrate into the capillary tube and form a "liquid slug". The "slug formation" is unique for capillary tubes, due to the typical high aspect ratios (length/diameter) of the tubes. In this past work, the authors balanced the dynamic pressure with the Laplace pressure at the bottom of the "slug" and found a critical impact velocity for this unique regime.

In the case of droplets impacting meshes, recent work ${ }^{35,36}$ have identified three impact regimes, namely "no penetration", "protrusion" and "complete penetration". The critical velocity $v_{\mathrm{c}}$ that divides no penetration from complete penetration is obtained by balancing the dynamic impact pressure $P_{\mathrm{d}} \propto \rho v_{\mathrm{c}}{ }^{2}$ and the capillary pressure $P_{\mathrm{c}} \sim \sigma \Gamma / A$, where $A$ and $\Gamma$ are the opening area and the perimeter of the mesh pore, thence $v_{\mathrm{c}}$ scales as $(\sigma \Gamma / A)^{1 / 2}$. The protrusion is a unique transition where a portion of the droplet extends beyond the mesh pores temporarily. Moreover, $v_{\mathrm{c}}$ was found to increase as the pore depth increases due to viscous dissipation. ${ }^{15}$ In the extreme penetration case, most of the droplet volume shoots out of the pores and forms liquid 'slugs' extending from the opposite surface of the mesh. A further study demonstrated that superhydrophobic meshes ${ }^{16}$ could effectively resist incoming droplets and eliminate protrusion. Droplet penetration in a superhydrophobic mesh is suppressed at a critical recoil velocity $v_{\mathrm{r}} \sim(\sigma / D)^{1 / 6}(\sigma \Gamma / A)^{1 / 3}$, indicating that penetration cannot be achieved by surface wettability alone. Additionally, 'pancake bouncing' was reported on superhydrophobic meshes. ${ }^{16,35}$

In another work, Bordoloi et al. ${ }^{37}$ studied the penetration of millimetre-sized water droplets ( $\sim 5 \mathrm{~mm}$ diameter $)$ through a millimetric pore while the entire system was submerged in an oil tank. A thin oil film was found to separate the wall of the pore from the droplet for pores with rounded edges. In this case the surface wettability of the pore wall was found to have no effect on the penetration dynamics. In contrast, for pores with sharp edges, the droplets contacted the pore wall immediately after the impact and the surface wettability affected the penetration dynamics. Joung and Buie ${ }^{38}$ investigated the impact of droplets on thin porous films (i.e. papers) and found four impact outcomes ranging from droplet sticking to the impact surface to splashing, all dependent on the We number. ${ }^{44}$ While these past studies have focused on various solid substrates, scarce evidence exists on the impact dynamics on textiles. In fact, past studies have often focused on static wetting. ${ }^{39,40}$ In industrial environments, liquid repellency of textiles has been sought after using hydrophobic coatings but little attention has been paid to the liquid dynamics or the textile pore size. In addition, studies focusing on the contact line and impact dynamics before droplet recoiling, or on the early times after the initial contact between a droplet and a textile, are scarce. 
In this paper, we provide experimental data on the impact dynamics before receding, characterising the simultaneous yet separate lateral spreading and normal penetration, and compare the results with that for impacting impermeable solids and metallic meshes. We also show that penetration is possible for even hydrophobic textiles, and that the most critical parameters are the pore size, and the speed of impact. We study the impact of millimetre-sized droplets on various textiles including those whose surface has been treated to be hydrophobic (water repellent). Our experiments used nylon mono-filament textiles with pore sizes ranging from 100 to $300 \mu \mathrm{m}$. Our textiles were chemically treated with fluorinated coatings based on $1 \mathrm{H}, 1 \mathrm{H}, 2 \mathrm{H}, 2 \mathrm{H}$ perfluorooctyl acrylate (PFAC6) and $1 H, 1 H, 2 H, 2 H$ perfluorodecyl acrylate (PFAC8). These fluorinated coatings are widely known for the hydrophobic effects when applied to textiles, papers and other substrates. ${ }^{41}$ Microscopy indicates that the textile geometry, as shown in Fig. 1 and 2, i.e. pore size $\left(d_{\text {mesh }}\right)$ and yarn radius $\left(R_{\text {yarn }}\right)$, is not affected by the chemical treatment. The hysteresis of the contact angle (advancing minus receding contact angle) on PFAC6 and PFAC8 films is reported to be $55^{\circ}$ and $37^{\circ}$, respectively. ${ }^{39}$ Equilibrium contact angles are both approximately $123^{\circ}$, and therefore clearly indicating the hydrophobic nature of the perfluorinated polymers. The non-treated nylon textile is hydrophilic and the equilibrium contact angle is approximately $43^{\circ}$.

In this work, the impact of droplets on textiles was recorded using a high-speed imaging system and the obtained images were later analyzed for the extraction of the impact speed, the initial droplet diameter, the spreading diameter and the height of the droplet as a function of the time after impact. The recordings



Fig. 1 Schematic diagram of a droplet impacting a textile substrate (not to scale). The textile is placed perpendicular to the $z$-axis at $z=0$, and the droplet moves along the $z$-axis.

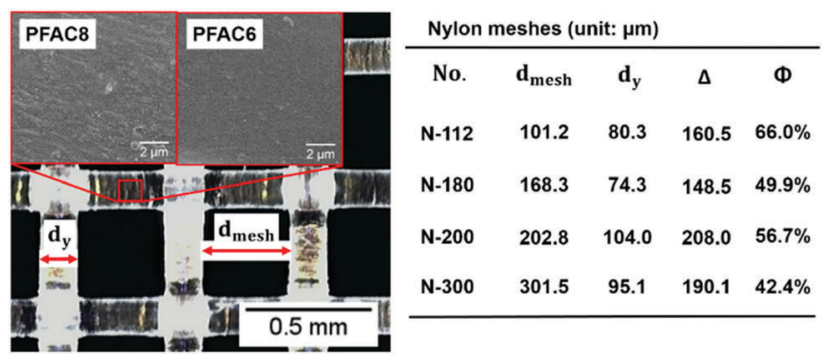

Fig. 2 (left) Optical microscopy image showing the geometry of a nylon mesh (textile), the upper insets show SEM images of the surface topography introduced by PFAC6 and PFAC 8 coatings. The scale bars in the SEM images correspond to $2 \mu \mathrm{m}$. (right) Table of pore size $\left(d_{\text {mesh }}\right)$, yarn diameter $\left(d_{\mathrm{y}}\right)$, mesh thickness $(\Delta)$, and solid fraction $(\phi)$ for all the meshes used in this paper. were also used to identify whether the droplet penetrated or stayed on the upper boundary of the textile. Our minimum optical resolution was $15.15 \mu \mathrm{m}$ per pixel.

\section{Experimental}

\section{Materials and methods}

In this paper, we study the impact of aqueous glycerol droplets on nylon textiles. The working fluid is a solution of distilled water and pure glycerol (Sigma-Aldrich, assay of purity $\geq 99.5 \%$, UK), with a measured surface tension of $\sigma \approx 68.5 \pm$ $1.5 \mathrm{mN} \mathrm{m}^{-1}$ at room temperature. The textiles consist of nylon monofilaments aligned and intertwined at right angles to one another, forming pore sizes ranging from $100 \mu \mathrm{m}$ to $300 \mu \mathrm{m}$. Textile surfaces were perfluorinated with PFAC6 and PFAC8 coatings using plasma treatment ${ }^{42}$ to render hydrophobicity. After the treatment, the textiles were cut into $20 \times 8 \mathrm{~mm}^{2}$ sections. For every impact experiment, a textile section was horizontally clamped at its ends on a Deßen micro-test device. Through this device we applied a constant tensile force of $2.5 \pm 0.1 \mathrm{~N}$. The tensile force minimised the deformation of the textile in the direction normal to the textile plane. No change on the pore size was observed due to this stretching.

A satellite-free droplet generator ${ }^{43,44}$ shown in Fig. 3, positioned right above the textile surface, was used to generate liquid droplets. In brief, the droplet generator consists of two parts: a loudspeaker and a liquid reservoir with a $2.0 \mathrm{~mm}$ diameter nozzle. The loudspeaker is used to send fast and short single pulses to generate millimetre-sized droplets. By adjusting the liquid meniscus position at the nozzle and controlling the pulse width and amplitude we could reliably control the speed of impact and size of droplets. We achieved a range of impact velocities within the range 0.88 to $1.90 \mathrm{~m} \mathrm{~s}^{-1}$. Droplet impact was visualised using a shadowgraphy system consisting of a high-speed camera (Phantom V710 with a Tamron SP AF60 macro lens), an optical diffuser, and a high-intensity illumination from an array of LEDS. Droplet impact was recorded at 64000 frames per second, a frame rate at least 3 times faster than previous relevant experiments. ${ }^{15,16,38}$ The exposure time was $10.0 \mu$ s for all experiments. Images were then analysed in ImageJ and Matlab. In all our experiments, the droplet size was kept constant at $D \approx 1.56 \pm 0.12 \mathrm{~mm}$.

\section{Results and discussion}

Various studies of droplets impacting porous substrates and meshes have been carried out in recent years focusing on the impact speed threshold for capture and penetration of a hole in a solid substrate, ${ }^{15}$ the droplet penetration speed in terms of pore size on porous films, ${ }^{38}$ and the droplet contact time on metallic meshes. ${ }^{16}$ In this paper, we study the radial spreading following the droplet impact on textiles and develop scaling arguments to obtain the penetration speed threshold in terms of the droplet diameters and the textile geometry.

Past studies on droplets impacting non-porous surfaces have identified four stages in the impact process, namely kinematic (1), 


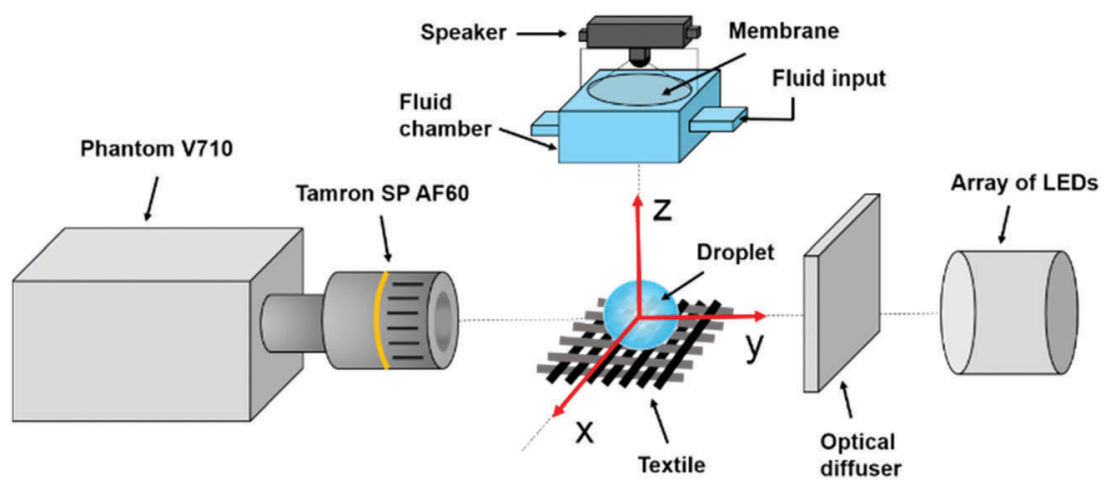

Fig. 3 Schematic diagram of the shadowgraphy apparatus.

spreading (2), relaxation (3) and wetting/equilibrium (4) stages. For impact on a porous textile, we have identified an additional stage, which we have named penetration (0), occurring at the earliest time after impact. Our results for later times show a quantitative agreement with the kinematic stage. The spreading stage is observed in all the tested textiles and jetting conditions. The dynamics of the droplet in stages 2 to 4 were also found to be in agreement with previous results. In our analysis, the spread factor is defined as $d^{*}=\frac{d}{d_{\max }}$, where $d_{\max }$ is the maximum spreading diameter, and $d$ is the spreading diameter at time $t$. The experimental data, shown in Fig. 4 and subsequent figures, is reported as a function of the dimensionless time $t^{*}=t\left(\frac{v}{D}\right)$.

For textiles with pore size between 100 and $300 \mu \mathrm{m}$ and We $>20$, in the penetration stage $\left(0<t^{*}<0.25\right)$ the droplet passes through the textile but its shape remains spherical. This feature is illustrated in the insets of Fig. 5, where circular red dashed curves have been over imposed onto the planar projection of the droplet shape. The portion of the droplet that penetrates through the textile pores forms liquid filaments that extend out of the textile from the back surface, as seen in Fig. 5 . The penetration stage has only been qualitatively characterized before on super-hydrophobic micro-grids. ${ }^{45}$ We can model the surface of the droplet during the penetration phase as a sphere of diameter $D$ translating with velocity $v$ in the negative $z$ direction. Fig. 6 shows the $x z$ projection of the droplet at two times: at the impact time $\left(t^{*}=0\right)$ and at a time $t$. For our experimental conditions, the shape of the droplet before impact and at early times after impact can be approximated as a circle. The centre of this circle is found at a distance $D / 2$ away from the surface, i.e. at $z\left(t^{*}=0\right)=\frac{D}{2}$. The equation of such a circle is thus:

$$
x^{2}+\left(z-\frac{D}{2}\right)^{2}=\left(\frac{D}{2}\right)^{2}
$$

Consequently, as the droplet moves along the $-z$ direction, the circular projection is given by:

$$
x^{2}+\left(z-\frac{D}{2}+v t\right)^{2}=\left(\frac{D}{2}\right)^{2}
$$

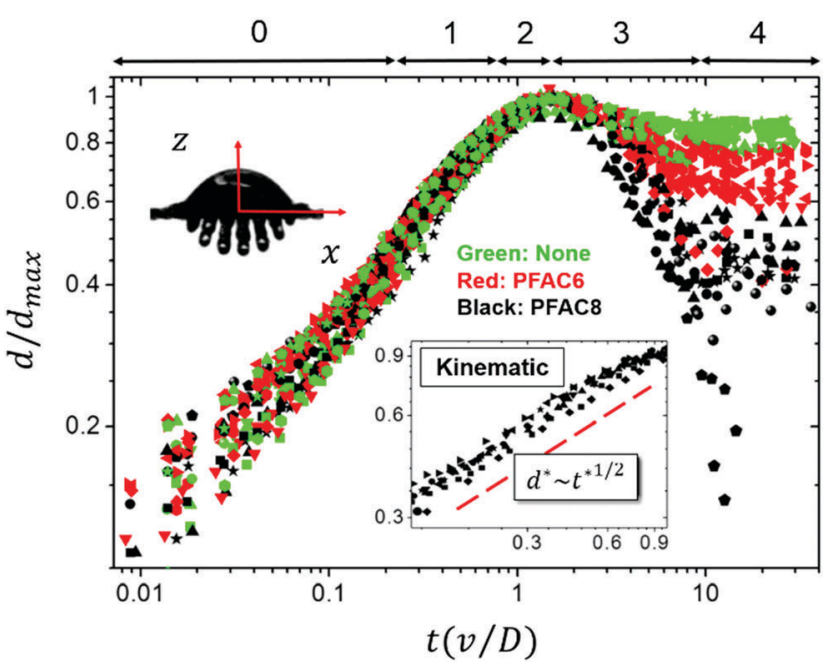

Fig. 4 Droplet spreading and receding diameters as a function of $t^{\star}=t(v / D)$, for different We numbers and various textiles with pores ranging from 100-300 $\mu \mathrm{m}$. The colours indicate different textile coatings: green for non-coated, red for PFAC6 coated, and black for PFAC8 coated textiles. The inset shows the scaling $d^{*} \sim t^{\star 1 / 2}$ characteristic of the kinematic stage. All data included. Measurement error \pm 2 pixels.

According to this model, the spreading diameter $d$ should be comparable to the length of the intersection of the $x z$ projection with the plane $z=0$,

$$
d=2 \sqrt{D v t-(v t)^{2}}
$$

and the droplet height should follow:

$$
h=D-v t
$$

The experimental data shown in Fig. 5, for $0<t^{*}<0.25$ and We $>20$, agrees well with the predictions of eqn (3) and (4). The textile pores allow the liquid to flow through, lifting the compressibility constrain for an impenetrable boundary predicted by Rioboo et al..$^{12}$ As a result, a portion of the droplet passes through the substrate without a visible change in shape. Moreover, droplet splashing on a flat impermeable substrate ${ }^{12}$ is accompanied by the formation of a thin gas layer between the droplet and the substrate. In our case, air can pass through the pores. 



Fig. 5 Droplet penetration at very early times. The results show the droplet penetrating the textile with no shape variations. Here, $d^{*}=\frac{d}{d_{\max }}$ and $h^{*}=\frac{h}{D}$. The legend indicates the textile mesh size in micrometres, the type of coating, and the We number, e.g. $300 \mathrm{C} 635$ stands for mesh size $300 \mu \mathrm{m}$, PFAC6 coating, and $W e \approx 35$. Penetration is not observed for We $<20$. The dashed lines correspond to eqn (3) (left) and eqn (4) (right). Measurement error \pm 2 pixels.
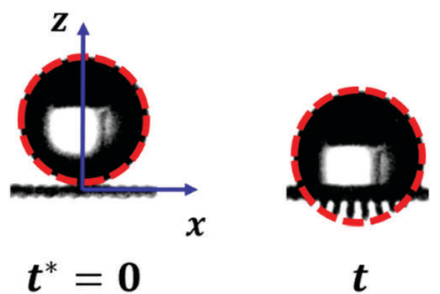

Fig. 6 Droplet impact on a textile at the impact point $\left(t^{\star}=0\right)$ and at a time $t$.

Eggers $e t ~ a l .{ }^{23}$ carried out simulations of a droplet impacting a flat, impermeable, solid substrate. Their simulations show that the re-direction of the fluid momentum by the boundary from the normal to the lateral direction during impact is due to the development of a high-pressure region at the base of the droplet. The redirection of the flow is accompanied by a rapid pressure decay. ${ }^{23}$ For conventional impermeable substrates, the non-dimensional time $t^{*}$ for the pressure decay to commence is no greater than $0.1{ }^{12,23}$ For textiles, and other substrates with high porosity, the impact-generated pressure is expected to be much smaller than that for impenetrable substrates. Only when the droplet has penetrated sufficiently, the average pressure near the textile is sufficient large to cause the flow re-direction. As a result, the re-direction process in textiles is retarded in comparison to what happens on continuous impermeable solids, permitting us to capture stage 0 by high-speed imaging. Physically, the penetration stage is characterised by the lack of a lamella. In Fig. 7, we contrast droplet shapes corresponding to stage $0\left(t^{*}<0.25\right)$ for the impact on a flat impermeable substrate and the impact on a textile. In the case of textiles, a liquid lamella does not form during the penetration phase and little lateral distortion of the droplet is seen while the droplet impacts and penetrates the textile. In fact, the formation of the lamella $\left(t^{*} \approx 0.25\right)$ coincides with the transition to the kinematic stage (1).

Surface tension effects arise at the pace given by the capillary time:

$$
t_{\text {capillary }}{ }^{*}=\left(\frac{\rho d_{\text {mesh }^{3}}{ }^{3}}{\sigma}\right)^{1 / 2} \frac{v}{D}
$$



Fig. 7 Image sequences comparing droplet impact on an N-300 textile and a liquid-repellent glass slide for $W e \approx 36$ and $W e \approx 40$, to illustrate the effect of substrate permeability on the droplet deformation. Both the glass substrate and the textile are coated with PFAC6.

In our experimental conditions $t_{\text {capillary }}{ }^{*}$ is in the range 0.1-0.7. This range coincides with the observed transition to the wellknown kinematic stage, identified by the $d^{*} \sim t^{*^{1 / 2}}$ scaling as plotted in Fig. 4. Note that, eqn (3) recovers $d^{*} \sim t^{* 1 / 2}$ for $t^{*}<0.1 .^{46}$ The kinematic stage was first proposed in the context of the impact of droplets on liquid reservoirs, ${ }^{47}$ and was subsequently adopted to describe the impact of droplets on non-porous solids. ${ }^{46}$ This stage is observed for all the We numbers studied here for $0.25<t^{*}<0.9$, and is characterized by the late formation of a radial liquid lamella at the base of the droplet. The lamella expands on the textile surface, while the droplet volume above the lamella flattens. The droplet then transitions to the known "spreading stage", where $d^{*}$ no longer linearly varies with $t^{1 / 2}$. In the "spreading stage", the droplet gradually flattens into a "pancake" shape and the contact line reaches its maximum expansion diameter $d_{\max }$. In this stage, the jetted liquid fingers on the back surface of the textile break up into secondary droplets, as will be shown in Fig. 9c.

The droplet dynamics during the spreading phase is observed for all the textiles investigated here, regardless of the We number. Such finding agrees with previous experimental data for impact on non-porous solids; this data shows that the temporal development of $d^{*}$ changes significantly only when We is at least two orders of magnitude larger than in the cases explored here. ${ }^{46}$ Furthermore, our experiments show that $d_{\max } \cong 2 D$. This result is also in agreement with literature results for nonporous solids. ${ }^{20}$ 


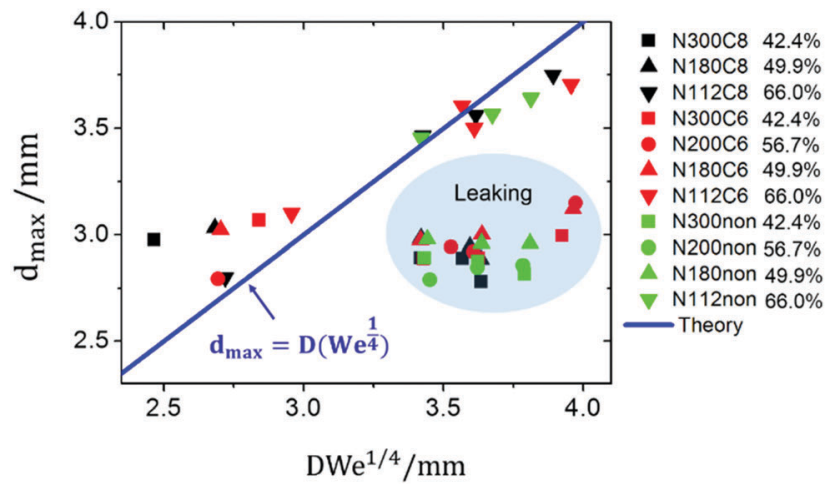

Fig. 8 Maximum spreading diameter, $d_{\text {max }}$, as a function of the expected scaling $d_{\max }=D W \mathrm{e}^{1 / 4}$. The blue-shaded region shows the cases where leaking (penetration) occurs. The various symbols indicate the substrates used, i.e. non-coated, PFAC6 and PFAC 8 coatings.

a

\section{b}

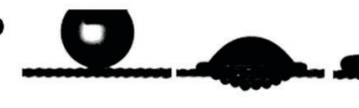

c

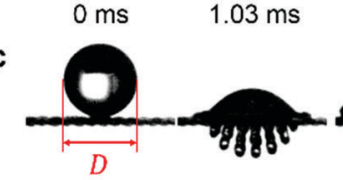

$0 \mathrm{~ms}$

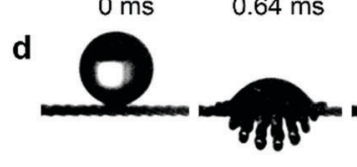

$0 \mathrm{~ms}$

$0.64 \mathrm{~ms}$

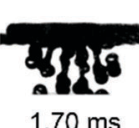

$1.70 \mathrm{~ms}$

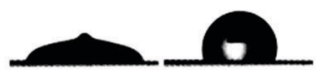

$6.17 \mathrm{~ms}$ Equilibrium

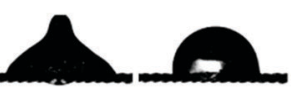

$6.17 \mathrm{~ms}$ Equilibrium

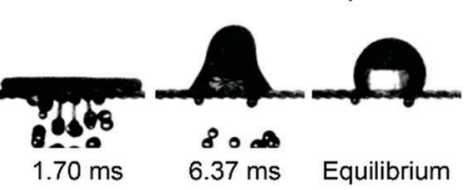

Fig. 9 Penetration behavior of droplet $(D \approx 1.56 \mathrm{~mm})$ for increasing We numbers. The sequence in (a) shows no penetration for We $\approx 8.9$ and $d_{\text {mesh }} \approx 101 \mu \mathrm{m}$. In (b), We $\approx 8.3$ and $d_{\text {mesh }} \approx 203 \mu \mathrm{m}$, a portion of the droplet penetrates the textile but is driven back to the top surface. In (c), We $\approx 35.0$ and $d_{\text {mesh }} \approx 303 \mu \mathrm{m}$, a fraction of the droplet permanently penetrates the textile, forming liquid filaments that break up into secondary droplets. The textiles for $(\mathrm{a}-\mathrm{c})$ are all PFAC8 nylon. In (d), We $\approx 25$ and $d_{\text {mesh }} \approx 303 \mu \mathrm{m}$ and the textile is non-coated nylon. The penetration behaviour of (d) is similar to (c) but the receding phase differs due to the hydrophilicity of the non-coated textile.

After reaching $d_{\max }$, the "pancake" recedes, as the impact dynamics enters the relaxation stage 3 . For non-porous substrates, this stage is known to be affected by the wettability of the substrate and contact angle hysteresis. ${ }^{48}$ Following stage 3 , a comparatively long equilibrium stage (stage 4) occurs where the droplet volume stops receding and oscillates in the $z$ direction. Eventually, the droplet finds a stable configuration. Fig. 4 shows that the rates at which the droplets recede are different for PFAC8 and PFAC6 textiles. Moreover, the equilibrium diameters for PFAC8 textiles are smaller. One can speculate that the smaller contact angle hysteresis ${ }^{41}$ of PFAC8 enables the contact line to move more freely.
Summarising, stages 1 to 4 are similar to those previously identified for impermeable solids ${ }^{46}$ whereas the penetration stage (stage 0), is unique to permeable substrates such as textiles. Visually, this stage is characterized by the lack of a liquid lamella at the droplet base.

As discussed, droplets impacting impermeable solids ${ }^{23,49}$ and some micro-textured substrates ${ }^{50}$ are compressed at the contact area, generating a high pressure field whose characteristic magnitude is of the $\operatorname{order}^{23} P(t) \sim \rho v^{2}\left(\frac{D}{2 v t}\right)^{1 / 2}$. It has been reported that the impact force on the solid substrate reaches its maximum $^{51}$ at $t_{\max } \sim D / 8 v$, resulting in a pressure ${ }^{23}$ $P\left(t_{\max }\right) \sim 2 \rho v^{2}$. This peak pressure is often referred to as "water hammer" $P_{\mathrm{WH}}$ and has been reported for the impact of droplets on non-wettable microtextured surfaces, ${ }^{50}$ microgrids ${ }^{45}$ and meshes. ${ }^{52}$ Essentially, $P_{\mathrm{WH}}$ is generated due to the temporary volume compression at the bottom of the droplet at the moment of impact and is alleviated by the droplet lateral spreading. ${ }^{50}$ For non-wettable meshes, $P_{\mathrm{WH}}$ has been found to scale as $k \rho v^{2}$, where $k$ is a scaling constant that depends on the mesh size. ${ }^{52}$ In our experiments, the textile porosity permits the penetration of the droplet and consequently the build-up pressure is lower than that found for solid substrates. In our experiments, the lamella is observed at much later times, so we claim the water hammer pressure does not contribute to the spreading dynamics in textiles.

The water hammer effect and the dynamic pressure have been found to follow the relationship: ${ }^{52}$

$$
P_{\mathrm{WH}}=a P_{\mathrm{D}},
$$

where $a$ is a scaling pre-factor. The water hammer effect becomes important when $a \geq 1$. Xu et $a l .{ }^{52}$ have found that $a$ is function of the number of pores $N$ covered by the droplet during the impact and experiments have shown that $a \geq 1$ for $N=\mathrm{O}(100)$. In our conditions, $N$ is of the order of 10 , therefore, the water hammer is expected to be negligible.

\section{Effect of mesh geometry on maximum spreading $\boldsymbol{d}_{\text {max }}$}

As discussed in the introduction, the maximum spreading diameter for droplets impacting super-hydrophobic solid surfaces ${ }^{20}$ and low porosity meshes, ${ }^{16}$ has been found to follow the scaling $d_{\text {max }} \propto D \cdot \mathrm{We}^{1 / 4}$. Our results, seen in Fig. 8, are consistent with this observation. The scaling $d_{\max } \propto D \cdot \mathrm{We}^{1 / 4}$, solid line in Fig. 8 with a numerical coefficient equal to 1 , is reasonably well followed by the data for densely packed textile fibres (solid fraction, $\phi>66 \%$ ), case for which penetration is not observed. In contrast, for cases showing penetration, the data does not adhere to the scaling. The experimental data, especially at relatively high We, shows significantly smaller values of $d_{\max }$ than those predicted by $d_{\max } \approx D \cdot \mathrm{We}^{1 / 4}$, highlighting the non-negligible effect of the liquid penetration into the textile on lateral spreading.

\section{Two regimes of droplet penetration}

Through visualisation, we identified two distinctive regimes of droplet penetration: partial and complete (Fig. 9b and c). The former regime shows that a fraction of the droplet volume 
passes through the textile structure and then mostly retracts back to the (upper) impact surface. In contrast, in complete penetration the droplet extends beyond the textile squirting liquid filaments which eventually break into secondary droplets. Moreover, we found that the penetration regime is observed for droplet impacting on both hydrophilic and hydrophobic textiles, Fig. 9. In the partial penetration regime, PFAC6 textiles do not drive the penetrated volume back to the upper textile surface, leaving a droplet stain (or footprint) visible at the back surface. In contrast droplets impacting PFAC8 textiles in the partial penetration regime entirely retract leave the back textile surface non-wetted. As before, we presume that such phenomena is owed to the differences in contact angle hysteresis between PFAC6 and PFAC8. Interestingly, the equilibrium configurations in Fig. 9a and c show a contact angle of $123 \pm 2^{\circ}$, despite having different impact dynamics. This observation confirms that evaluating liquid repellency solely on the equilibrium contact angle $\mathrm{e}^{53}$ is clearly inappropriate, particularly for high-speed impact. A possible further study could explore the behavior of the dynamic contact angle in terms of the textile characteristics.

\section{Critical pore size for droplet penetration}

It has been found that, viscous effects associated with the flow of liquid through a single pore play a minor role in resisting penetration, when the Reynolds number $\operatorname{Re}=\frac{\rho v D}{2 \mu}>10$, where $\eta$ is the dynamic viscosity. ${ }^{15}$ In our experiments, $\rho \approx 10^{3} \mathrm{~kg} \mathrm{~m}^{-3}$, $\mu \sim 10^{-2}$ Pa s, the typical impact velocity is $v_{\text {typical }} \sim 1 \mathrm{~m} \mathrm{~s}^{-1}$, and the characteristic pore size is $d_{\text {mesh }} \sim 100 \mu \mathrm{m}$. Re $\sim 10$, indicating that viscous effects are relatively unimportant in the impact regimes studied here. The capillary force exerted by the mesh pore ${ }^{35}$ scales as $\sigma d_{\text {mesh }}$, consequently, from the balance of fluid inertia and capillary forces, we deduce:

$$
k \rho v^{2} \approx \frac{\sigma}{d_{\text {mesh }}}
$$

where $k$ is a scaling constant determined by the geometry of the textile mesh and potentially by the surface wettability. By using the Weber number, $\mathrm{We}=\frac{\rho v^{2} D}{2 \sigma}$, we obtain:

$$
d_{\text {mesh }} \approx \frac{D}{2 k \mathrm{We}}
$$

at the onset of penetration.

Based on eqn (8), we developed a penetration criterion based on the impact condition (Weber number) and the mesh size. Fig. 10 shows our experimental results for droplets impacting textiles for $7<\mathrm{We}<37$. The results in this figure are classified based on the penetration outcome, i.e. penetration or nopenetration (hollow or solid symbols).

Fig. 10 shows a clear separation of behavior in terms of the We number and the pore size. Eqn (8) seems to describe well the boundary between the penetration and no-penetration regions for $k \approx 0.24$ (this scaling constant is determined when both $D$ and $d_{\mathrm{m}}$ are using the standard units: $\mathrm{m}$ ). Our results are consistent with previous results ${ }^{16,35}$ obtained for hydrophobic and superhydrophobic copper meshes. For example, Ryu et al. ${ }^{35}$

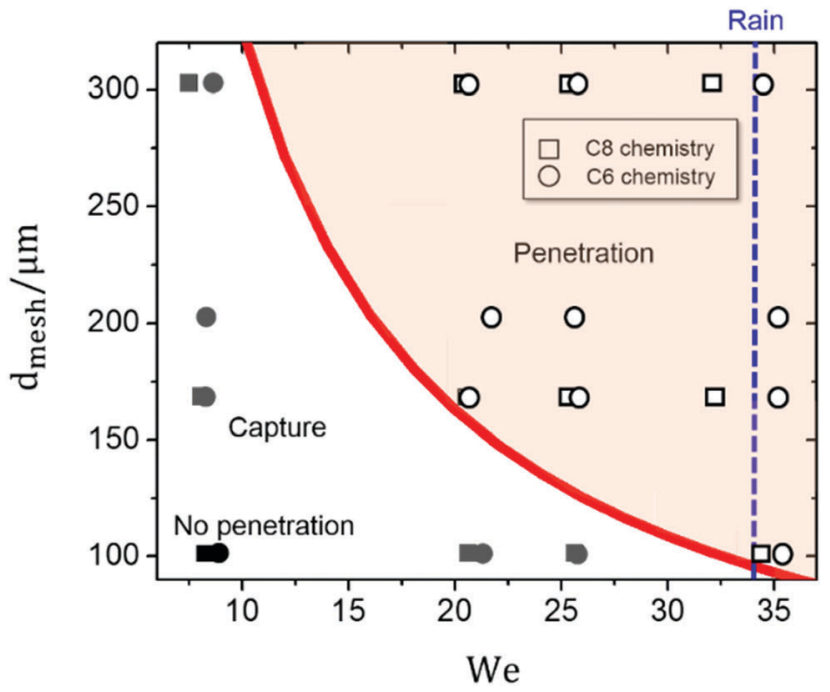

Fig. 10 Penetration regimes for droplets impacting textiles. The solid line indicates the boundary between the penetration and no penetration regimes. Hollow symbols denote textile penetration. Gray symbols indicate capture, i.e. a portion of the droplet penetrates but is eventually drawn back to the impact surface. Black symbols indicate no penetration where neither temporary liquid penetration nor droplet footprint is observed on the back surface.

showed that the onset of impact penetration for both hydrophobic and superhydrophobic meshes with mesh sizes $d_{\text {mesh }}>$ $178 \mu \mathrm{m}$ and $3.5 \mathrm{~mm}$ water droplets, occurs for $v \sim 1 \mathrm{~m} \mathrm{~s}^{-1}$, i.e. We $\approx 35$. Kumar et al. ${ }^{16}$ observed penetration for meshes with $d_{\mathrm{mesh}} \approx 140$ and $280 \mu \mathrm{m}$ at $\mathrm{We} \approx 35$ and 204.

The design and manufacture of modern raincoats, umbrellas and other general clothing include the use of hydrophobic woven textiles aiming to repel water while maintaining breathability. Our results indicate the contrary to popular belief; water repellency cannot be achieved by adjusting the fabric hydrophobic characteristics alone, as sufficiently rapid drops could penetrate a fabric regardless of its surface properties. A useful example to discuss is rain. A raindrop of a size $d_{\text {rain }} \sim 1.6 \mathrm{~mm}^{54}$ impacting at a speed of $v_{\text {rain }} \cong 1.77 \mathrm{~m} \mathrm{~s}^{-1}$ penetrates any hydrophobic fabric with a pore size above $100 \mu \mathrm{m}$. A faster or a larger droplet will require a smaller pore to be repelled.

\section{Conclusions}

In this manuscript, high-speed imaging was used to investigate the droplet impact dynamics on textiles having varying pore sizes and two different hydrophobic and one hydrophilic wettabilities. A key insight into the impact dynamics is the identification of the penetration stage, corresponding to $0<t^{*}<0.2$. During this stage, the droplet penetrates the textile through the textile pores and forms liquid filaments under the textile surface. Interestingly, in this stage, the droplet shape above the textile is not perturbed by the impact and no lamella is observed. Our findings are supported by previous experimental results on metallic meshes. ${ }^{16,35}$

The maximum spreading diameter $d_{\max }$ has been found to conform to the well-known scaling $d_{\max }=D\left(\mathrm{We}^{1 / 4}\right)$, which was 
initially developed for impermeable solids, for textiles with a solid fraction $\phi>66 \%$. This conclusion is in close agreement with the results by Kumar et al. ${ }^{16}(\phi>70 \%)$ on superhydrophobic meshes.

We have shown three regimes of droplet impact penetration on non-wettable meshes, namely 'no penetration', 'capture' and 'complete penetration'. Balancing the dynamic impact pressure $\left(\sim \rho v^{2}\right)$ and the capillary pressure $\left(\sim \sigma / d_{\text {mesh }}\right)$, the critical pore size has the form $d_{\text {mesh }} \sim D$ /We. This simple model and our parametric experimental studies indicate that reducing the mesh size is an effective way to avoid liquid capture and penetration. This result is critical for the design of textiles aiming to avoid penetration hazardous liquids.

\section{Conflicts of interest}

There are no conflicts to declare.

\section{Acknowledgements}

The authors acknowledge Prof. Alfonso A. Castrejón-Pita, of the University of Oxford, for his technical assistance. Prof. Asa H. Barber of London South Bank University is acknowledged for useful discussions and background work. This work has been financially supported by the Material Research Institute (MRI) of Queen Mary University of London, and the UK Defence Science \& Technology Laboratory (Dstl). L. B. acknowledges ERC Starting Grant FlexNanoFlow (no. 715475). M. A. Q.-S. acknowledges the funding from the Mexican Energy Ministry (SENER) and the National Council for Science and Technology (CONACyT).

\section{References}

1 W. H. Wischmeier and D. D. Smith, Trans., Am. Geophys. Union, 1958, 39, 285-291.

2 Y. Liu, M. Andrew, J. Li, J. M. Yeomans and Z. Wang, Nat. Commun., 2015, 6, 10034.

3 K. Liu and L. Jiang, Annu. Rev. Mater. Res., 2012, 42, 231-263.

4 R. Blossey, Nat. Mater., 2003, 2, 301-306.

5 L. Mishchenko, B. Hatton, V. Bahadur, J. A. Taylor, T. Krupenkin and J. Aizenberg, ACS Nano, 2010, 4, 7699-7707.

6 A. J. Meuler, G. H. McKinley and R. E. Cohen, ACS Nano, 2010, 4, 7048-7052.

7 C. Cao, M. Ge, J. Huang, S. Li, S. Deng, S. Zhang, Z. Chen, K. Zhang, S. S. Al-Deyab and Y. Lai, J. Mater. Chem. A, 2016, 4, 12179-12187.

8 S. A. Brewer and C. R. Willis, Appl. Surf. Sci., 2008, 254, 6450-6454.

9 I. V. Roisman, R. Rioboo and C. Tropea, Proc. R. Soc. London, Ser. A, 2002, 450, 1411-1430.

10 M. Rein, Fluid Dyn. Res., 1993, 12, 61-93.

11 A. L. Yarin, Annu. Rev. Fluid Mech., 2006, 38, 159-192.

12 R. Rioboo, M. Marengo and C. Tropea, Exp. Fluids, 2002, 33, 112-124.
13 Y. Liu, L. Moevius, X. Xu, T. Qian, J. M. Yeomans and Z. Wang, Nat. Phys., 2014, 10, 515-519.

14 J. C. Bird, R. Dhiman, H.-M. Kwon and K. K. Varanasi, Nature, 2013, 503, 385-388.

15 É. Lorenceau and D. Quéré, J. Colloid Interface Sci., 2003, 263, 244-249.

16 A. Kumar, A. Tripathy, Y. Nam, C. Lee and P. Sen, Soft Matter, 2018, 14, 1571-1580.

17 E. M. Williams, M. Dodds, M. C. Taylor, J. Li and S. Michielsen, Forensic Sci. Int., 2016, 262, 66-72.

18 O. G. Engel, J. Res. Natl. Bur. Stand., 1955, 54, 281-298.

19 J. Marston, J. E. Sprittles, Y. Zhu, E. Li, I. U. Vakarelski and S. T. Thoroddsen, Powder Technol., 2013, 239, 128-136.

20 C. Clanet, C. Béguin, D. Richard and D. Quéré, J. Fluid Mech., 2004, 517, 199-208.

21 J. Lee, N. Laan, K. de Bruin, G. Skantzaris, N. Shahidzadeh, D. Derome, J. Carmeliet and D. Bonn, J. Fluid Mech., 2016, 786, R4.

22 N. Laan, K. G. de Bruin, D. Bartolo, C. Josserand and D. Bonn, Phys. Rev. Appl., 2014, 2, 044018.

23 J. Eggers, M. A. Fontelos, C. Josserand and S. Zaleski, Phys. Fluids, 2010, 22, 062101.

24 M. Pasandideh-Fard, Y. Qiao, S. Chandra and J. Mostaghimi, Phys. Fluids, 1996, 8, 650-659.

25 T. Mao, D. Kuhn and H. Tran, AIChE J., 1997, 43, 2169-2179.

26 C. Antonini, A. Amirfazli and M. Marengo, Phys. Fluids, 2012, 24, 102104.

27 B. L. Scheller and D. W. Bousfield, AIChE J., 1995, 41, 1357-1367.

28 A. Gauthier, S. Symon, C. Clanet and D. Quéré, Nat. Commun., 2015, 6, 8001.

29 P. Tsai, S. Pacheco, C. Pirat, L. Lefferts and D. Lohse, Langmuir, 2009, 25, 12293-12298.

30 A. Lafuma and D. Quéré, Nat. Mater., 2003, 2, 457-460.

31 D. Bartolo, F. Bouamrirene, E. Verneuil, A. Buguin, P. Silberzan and S. Moulinet, EPL, 2006, 74, 299.

32 M. Reyssat, A. Pépin, F. Marty, Y. Chen and D. Quéré, EPL, 2006, 74, 306.

33 J. Bico, U. Thiele and D. Quéré, Colloids Surf., A, 2002, 206, 41-46.

34 A. Delbos, E. Lorenceau and O. Pitois, J. Colloid Interface Sci., 2010, 341, 171-177.

35 S. Ryu, P. Sen, Y. Nam and C. Lee, Phys. Rev. Lett., 2017, 118, 014501.

36 H. Ghadiri and D. Payne, Eur. J. Soil Sci., 1981, 32, 41-49.

37 A. D. Bordoloi and E. K. Longmire, J. Fluid Mech., 2014, 759, 520-545.

38 Y. S. Joung and C. R. Buie, Phys. Rev. E: Stat., Nonlinear, Soft Matter Phys., 2014, 89, 013015.

39 G. Zhang, R. Parwani, C. A. Stone, A. H. Barber and L. Botto, Langmuir, 2017, 12072-12079.

40 E. Kissa, J. Colloid Interface Sci., 1981, 83, 265-272.

41 Fluorinated polymers: Volume 1: Synthesis, Properties, Processing and Simulation, ed. B. Ameduri and H. Sawada, Royal Society of Chemistry, 2016.

42 S. Coulson, I. Woodward, J. Badyal, S. Brewer and C. Willis, J. Phys. Chem. B, 2000, 104, 8836-8840. 
43 J. Castrejón-Pita, G. Martin, S. Hoath and I. Hutchings, Rev. Sci. Instrum., 2008, 79, 075108.

44 J. Castrejón-Pita, N. Morrison, O. Harlen, G. Martin and I. Hutchings, Phys. Rev. E: Stat., Nonlinear, Soft Matter Phys., 2011, 83, 036306.

45 P. Brunet, F. Lapierre, F. Zoueshtiagh, V. Thomy and A. Merlen, Appl. Phys. Lett., 2009, 95, 254102.

46 R. Rioboo, M. Marengo and C. Tropea, Exp. Fluids, 2002, 33, 112-124.

47 M. Vollmer and K.-P. Möllmann, Phys. Educ., 2012, 47, 664.

48 C. Antonini, F. Villa, I. Bernagozzi, A. Amirfazli and M. Marengo, Langmuir, 2013, 29, 16045-16050.
49 D. Roux and J. Cooper-White, J. Colloid Interface Sci., 2004, 277, 424-436.

50 T. Deng, K. K. Varanasi, M. Hsu, N. Bhate, C. Keimel, J. Stein and M. Blohm, Appl. Phys. Lett., 2009, 94, 133109.

51 D. Soto, A. B. De Lariviere, X. Boutillon, C. Clanet and D. Quéré, Soft Matter, 2014, 10, 4929-4934.

52 J. Xu, J. Xie, X. He, Y. Cheng and Q. Liu, Exp. Therm. Fluid Sci., 2017, 82, 83-93.

53 A. Tuteja, W. Choi, M. Ma, J. M. Mabry, S. A. Mazzella, G. C. Rutledge, G. H. McKinley and R. E. Cohen, Science, 2007, 318, 1618-1622.

54 E. Villermaux and B. Bossa, Nat. Phys., 2009, 5, 697-702. 